\title{
Deformation of a magnetic liquid drop in an applied non-stationary uniform magnetic field
}

\author{
Alexander Tyatyushkin ${ }^{1, \star}$ \\ ${ }^{1}$ Institute of Mechanics, Moscow State University, Michurinskiy Ave., 1, Moscow 119192, Russia
}

\begin{abstract}
Small steady-state deformational oscillations of a drop of magnetic liquid in a nonstationary uniform magnetic field are theoretically investigated. The drop is suspended in another magnetic liquid immiscible with the former. The Reynolds number is so small that the inertia can be neglected. The variation of the magnetic field is so slow that the quasi-stationary approximation for the magnetic field and the quasi-steady approximation for the flow may be used.
\end{abstract}

\section{Introduction}

A non-stationary magnetic field applied to a drop of a magnetic liquid suspended in an ordinary liquid or to a drop of an ordinary liquid suspended in a magnetic liquid causes a motion of the liquids inside and outside the drop. Investigation of this phenomenon is interesting from the point of view of application to the actuation of motion of liquids in various devices including microfluidic ones. Besides, the study of this phenomenon is interesting from the point of view of basic science.

Results of experimental investigation of the behavior of magnetic liquid drops are presented in a number of works (see [1] for the references). For the theoretical description of the behavior of a magnetic liquid drop, models based on rather strong assumptions about the shape of the drop and about the flow inside and outside it were used [1]. The goal of the present work is to solve the problem about the shape of the drop and flow inside and outside it without the use of similar assumptions. Within this approach, the shape of the drop is determined by equations obtained with the help of asymptotic methods from the system of equations and boundary conditions that determine the magnetic field and the flow. In order to provide the applicability of the asymptotic methods, the scope of the present theoretical investigation is restricted by the case of weakly deformed drops.

\section{Setting of the problem}

\subsection{Object of investigation}

Consider a drop of incompressible magnetic liquid in an applied non-stationary uniform magnetic field with

^e-mail: tan@imec.msu.ru intensity $\vec{H}_{\mathrm{a}}=\vec{H}_{\mathrm{a}}(t)$, where $t$ is the time. The viscosity and magnetic permeability of the liquid inside the drop are $\eta_{\mathrm{i}}$ and $\mu_{\mathrm{i}}$. The radius of the drop in the undeformed state is $a$. The drop is suspended in an incompressible magnetic liquid with the viscosity $\eta_{\mathrm{e}}$ and magnetic permeability $\mu_{\mathrm{e}}$. The surface tension of the interface between the liquids is $\sigma_{\mathrm{s}}$. The liquids are regarded as sufficiently viscous for the small Reynolds number approximation to be valid. The variation of the magnetic field is regarded as sufficiently slow and the conductivities of the liquids are regarded as sufficiently small for the magnetic field due to the variation of magnetic field and the electric current in the liquid can be neglected, i.e., for the approximation of ferrohydrodynamics to be valid (see [2]).

\subsection{System of equations}

In order to find the drop shape, magnetic field intensity, $\vec{H}$, velocity, $\vec{v}$, and pressure, $p$, as functions of the radius-vector, $\vec{r}$, and time, $t$, under the above made assumptions, the system of equations of ferrohydrodynamic written down for magnetic fields in the quasi-stationary approximation and for flows in the quasi-steady approximation is used. This system consists of the continuity equation for an incompressible liquid

$$
\nabla \cdot \vec{v}=0
$$

the motion equation in the small Reynolds number and quasi-steady flow approximations

$$
\nabla \cdot\left(-p \hat{I}+\hat{\sigma}_{\mathrm{m}}^{\mathrm{T}}+\hat{\sigma}_{\mathrm{v}}\right)=0
$$

the Maxwell's equations in the ferrohyrodynamics and quasi-stationary field approximations

$$
\nabla \cdot \vec{B}=0, \quad \nabla \times \vec{H}=0,
$$


and the constitutive relation

$$
\vec{B}=\mu \vec{H} .
$$

Here, the formulas are written down for Gaussian system of units, $\cdot$ and $\times$ denote the scalar and vector products, $\vec{B}$ is the magnetic induction, $\eta=\eta_{\mathrm{i}}$ and $\mu=\mu_{\mathrm{i}}$ inside the drop, $\eta=\eta_{\mathrm{e}}$ and $\mu=\mu_{\mathrm{e}}$ outside it, $\nabla$ is the nabla operator, $\hat{I}$ is the identity tensor, $\hat{\sigma}_{\mathrm{m}}$ and $\hat{\sigma}_{\mathrm{v}}$ are the tensors of magnetic and viscous stresses expressed as follows

$$
\begin{aligned}
\hat{\sigma}_{\mathrm{m}} & =\frac{1}{4 \pi} \vec{H} \vec{B}-\frac{1}{8 \pi} \vec{B} \cdot \vec{H} \hat{I}, \\
\hat{\sigma}_{\mathrm{v}} & =2 \eta(\nabla \vec{v})^{\mathrm{S}},
\end{aligned}
$$

where $\vec{A} \vec{B}$ denotes the dyadic product of the vectors $\vec{A}$ and $\vec{B}, \nabla \vec{f}$ denotes the dyadic product of the nabla operator and the vector field $\vec{f}=\vec{f}(\vec{r}), \hat{T}^{\mathrm{T}}$ denotes the transpose of the tensor $\hat{T}, \hat{T}^{\mathrm{S}}=\frac{1}{2} \hat{T}+\frac{1}{2} \hat{T}^{\mathrm{T}}$ denotes the symmetric part of the second order tensor $\hat{T}$.

Using the continuity equation Eq. (1) and Maxwell's equations Eq. (3) and taking into account that the magnetic permeability is uniform, the motion equation Eq. (2) can be rewritten (see, e.g., [2]) in the form of the Navier-Stokes equation in the small Reynolds number approximation

$$
-\nabla p+\eta \Delta \vec{v}=0,
$$

where $\Delta$ is the Laplacian.

\subsection{Boundary conditions}

The boundary conditions on the interface between the liquids include the impenetrability condition

$$
\left.\vec{v}\right|_{\mathrm{e}} \cdot \vec{n}=\left.\vec{v}\right|_{\mathrm{i}} \cdot \vec{n}=v_{\mathrm{s} n},
$$

the no-slip condition

$$
[\vec{v}]_{\mathrm{S}} \times \vec{n}=0,
$$

the conditions for the jumps of the normal and tangential components of the stress vector $\vec{\sigma}_{n}=\vec{n}$.

$$
\begin{aligned}
\left(-p \hat{I}+\hat{\sigma}_{\mathrm{m}}+\hat{\sigma}_{\mathrm{v}}\right) & \\
\vec{n} \cdot\left[-p \hat{I}+\hat{\sigma}_{\mathrm{m}}+\hat{\sigma}_{\mathrm{v}}\right]_{\mathrm{s}} \cdot \vec{n} & =-\sigma_{\mathrm{s}} \mathcal{H}, \\
\vec{n} \cdot\left[\hat{\sigma}_{\mathrm{m}}+\hat{\sigma}_{\mathrm{v}}\right]_{\mathrm{s}} \times \vec{n} & =0,
\end{aligned}
$$

the continuity conditions for the tangential component of the magnetic field intensity

$$
[\vec{H}]_{\mathrm{S}} \times \vec{n}=0
$$

and for the normal component of the magnetic field induction

$$
[\vec{B}]_{\mathrm{S}} \cdot \vec{n}=0 .
$$

Here, $\left.A\right|_{\mathrm{i}}$ and $\left.A\right|_{\mathrm{e}}$ denote the values of the quantity $A$ on the interface between the liquids approached from inside and outside the drop, respectively, $[A]_{\mathrm{s}}=\left.A\right|_{\mathrm{e}}-$
$\left.A\right|_{\mathrm{i}}$ denotes the jump of the quantity $A$ at the interface when moving from the inside to the outside, $\vec{n}$ is the external normal unit vector at a given point of the interface, $v_{\mathrm{s} n}$ is the normal component of the velocity of the surface of the drop at a given point, $\mathcal{H}$ is the mean curvature at a given point of the surface of the drop. Note that the definition of the mean curvature used in the present work is such that it takes negative values on the surface of a convex domain.

The continuity conditions for the normal component of the magnetic induction Eq. (13) and for the tangential component of the magnetic field intensity Eq. (12) allow determining the following scalar and vector fields defined on the surface of the drop

$$
\begin{gathered}
B_{\mathrm{s} n}=\left.\vec{B}\right|_{\mathrm{i}} \cdot \vec{n}=\left.\vec{B}\right|_{\mathrm{e}} \cdot \vec{n}, \\
\vec{H}_{\mathrm{s} \tau}=\vec{n} \times\left(\left.\vec{H}\right|_{\mathrm{i}} \times \vec{n}\right)=\vec{n} \times\left(\left.\vec{H}\right|_{\mathrm{e}} \times \vec{n}\right) .
\end{gathered}
$$

Using the boundary conditions Eq. (12) and Eq. (13), the constitutive relation Eq. (4), and the introduced notations Eq. (14) and Eq. (15), one can transform the conditions for the stress vector Eq. (10) and Eq. (11) into the form

$$
\begin{gathered}
-[p]_{\mathrm{s}}+\frac{1}{8 \pi}\left(\frac{1}{\mu_{\mathrm{e}}}-\frac{1}{\mu_{\mathrm{i}}}\right) B_{\mathrm{s} n}^{2}-\frac{1}{8 \pi}\left(\mu_{\mathrm{e}}-\mu_{\mathrm{i}}\right) H_{\mathrm{s} \tau}^{2} \\
+2 \vec{n} \cdot[\eta \nabla \vec{v}]_{\mathrm{s}} \cdot \vec{n}=-\sigma_{\mathrm{s}} \mathcal{H} \\
\vec{n} \times\left([\eta \nabla \vec{v}]_{\mathrm{s}} \cdot \vec{n}+\vec{n} \cdot[\eta \nabla \vec{v}]_{\mathrm{s}}\right)=0 .
\end{gathered}
$$

The boundary conditions at infinity have the form

$$
\begin{array}{ll}
\vec{v} \rightarrow 0 & \text { as } r \rightarrow \infty, \\
p \rightarrow p_{\infty} & \text { as } r \rightarrow \infty, \\
\vec{H} \rightarrow \vec{H}_{\mathrm{a}} & \text { as } \quad r \rightarrow \infty,
\end{array}
$$

where $p_{\infty}$ is the pressure at infinity.

Besides, $\vec{v}(\vec{r}, t), p(\vec{r}, t)$, and $\vec{H}(\vec{r}, t)$ should be bounded for all the bounded values of $\vec{r}$.

\subsection{Deformation of the drop}

Let the surface of the drop be given by the following equation

$$
r=|\vec{r}|=a+h\left(\frac{\vec{r}}{r}, t\right) .
$$

The function $h(\vec{r} / r, t)$ can be represented in the form

$$
h=h\left(\frac{\vec{r}}{r}, t\right)=\sum_{n=2}^{\infty} \hat{h}_{n} \cdot \frac{n}{r^{n}},
$$

which excludes displacements of the drop by some vector without variation of its shape. Here, $\hat{h}_{n}=\hat{h}_{n}(t)$ $(n>1)$ are some tensors each of which is an arbitrary irreducible tensor of $n$th order, i.e., a tensor of $n$th order symmetric with respect to any pair of indices and such that its contraction with the identity tensor over any pair of indices is equal to zero (see [3]). Here and in what follows, $\vec{b}^{n}$ denotes $n$th dyadic degree of the vector $\vec{b}$ (i.e., the $(n-1)$-multiple dyadic product of the vector $\vec{b}$ by itself), ${ }^{n} \cdot$ denotes $n$-multiple contraction of tensors over the adjacent indices. 


\section{Solution}

\subsection{Magnetic field}

The intensity of the magnetic field is sought for in the form

$$
\vec{H}=\nabla \psi,
$$

where

$$
\psi=\left\{\begin{array}{l}
\vec{H}_{\mathrm{a}} \cdot \vec{r}-\sum_{n=1}^{\infty} \hat{M}_{\mathrm{e} n}{ }^{n} \cdot \frac{\vec{r}^{n}}{r^{2 n+1}}, r>a+h, \\
\vec{H}_{\mathrm{a}} \cdot \vec{r}-\sum_{n=1}^{\infty} \hat{M}_{\mathrm{i} n} \cdot \frac{n}{a^{2 n+1}}, r \leqslant a+h .
\end{array}\right.
$$

Here, $\hat{M}_{\mathrm{e} n}=\hat{M}_{\mathrm{e} n}(t)$ and $\hat{M}_{\mathrm{i} n}=\hat{M}_{\mathrm{i} n}(t)$ are tensors which are either some vectors for $n=1$ or, for $n>$ 1 , some irreducible tensors of $n$th order. For a field given in this form, Maxwell's equations Eq. (3), the constitutive relation Eq. (4), the condition at infinity Eq. (20), and the condition of boundedness of $\vec{H}$ are automatically satisfied.

\subsection{Flow}

The velocity and pressure in the flow are sought for in the form which can be constructed from general Lamb's solution (cf. [4], art. 336) for a quasi-steady flow

$$
\begin{aligned}
& \vec{v}=\left\{\begin{array}{l}
\vec{v}_{\mathrm{e}}, r>a+h, \\
\vec{v}_{\mathrm{i}}, r \leqslant a+h,
\end{array} \quad p=\left\{\begin{array}{l}
p_{\mathrm{e}}, r>a+h, \\
p_{\mathrm{i}}, r<a+h
\end{array}\right.\right. \\
& \vec{v}_{\mathrm{e}}=\sum_{n=1}^{\infty}\left[(2 n-1) r^{2}-(2 n+1) a^{2}\right] a^{n} \hat{B}_{\mathrm{e} n} \cdot \frac{n}{r^{n+1}} \\
& +\sum_{n=1}^{\infty}\left[(2 n-1) n r^{2}-(n-2) a^{2}\right] a^{n} \hat{C}_{\mathrm{e} n} \cdot \frac{\vec{r}^{n+1}}{r^{2 n+3}} \\
& +\sum_{n=1}^{\infty}\left[(-n+2) r^{2}+n a^{2}\right] a^{n} \hat{B}_{\mathrm{e} n}^{n-1} \cdot \frac{\vec{r}^{n-1}}{r^{2 n+1}} \\
& -\sum_{n=1}^{\infty}(n-2) n\left(r^{2}-a^{2}\right) a^{n} \hat{C}_{\mathrm{e} n}{ }^{n-1} \cdot \frac{\vec{r}^{n-1}}{r^{2 n+1}} \\
& +\sum_{n=1}^{\infty} n\left[\hat{\Omega}_{\mathrm{e} n} \stackrel{n-1}{\cdot} \frac{\vec{r}^{n-1} a^{n+2}}{r^{2 n+1}}\right] \times \vec{r}, \\
& \vec{v}_{\mathrm{i}}=-\sum_{n=1}^{\infty} 2 \hat{B}_{\mathrm{i} n} \cdot \frac{\vec{r}^{n+1}}{a^{n+1}}+\sum_{n=1}^{\infty} 2(n+1) \hat{C}_{\mathrm{i} n} \cdot \frac{n}{a^{n+1}} \\
& +\sum_{n=1}^{\infty}\left[(n+3) r^{2}-(n+1) a^{2}\right] \hat{B}_{\mathrm{i} n}^{n-1} \cdot \frac{\vec{r}^{n-1}}{a^{n+1}} \\
& -\sum_{n=1}^{\infty}(n+1)(n+3)\left(r^{2}-a^{2}\right) \hat{C}_{\mathrm{i} n}{ }^{n-1} \cdot \frac{\vec{r}^{n-1}}{a^{n+1}} \\
& +\sum_{n=1}^{\infty} n\left[\hat{\Omega}_{\mathrm{i} n}^{n-1} \cdot \frac{\vec{r}^{n-1}}{a^{n-1}}\right] \times \vec{r}
\end{aligned}
$$

$$
p_{\mathrm{e}}=\eta_{\mathrm{e}} \sum_{n=1}^{\infty} 2(2 n-1)\left(\hat{B}_{\mathrm{e} n}+n \hat{C}_{\mathrm{e} n}\right) \cdot \frac{n}{r^{n} a^{n}}+p_{\infty}
$$

$$
\begin{aligned}
p_{\mathrm{i}}=\eta_{\mathrm{i}} \sum_{n=1}^{\infty} & \frac{(2 n+2)(2 n+3)}{n} \\
& \times\left[\hat{B}_{\mathrm{i} n}-(n+1) \hat{C}_{\mathrm{i} n}\right]^{n} \cdot \frac{\vec{r}^{n}}{a^{n+1}}+p_{0} .
\end{aligned}
$$

Here, $\hat{\Omega}_{\mathrm{e} n}=\hat{\Omega}_{\mathrm{e} n}(t), \hat{B}_{\mathrm{e} n}=\hat{B}_{\mathrm{e} n}(t), \hat{C}_{\mathrm{e} n}=\hat{C}_{\mathrm{e} n}(t)$, $\hat{\Omega}_{\mathrm{i} n}=\hat{\Omega}_{\mathrm{i} n}(t), \hat{B}_{\mathrm{i} n}=\hat{B}_{\mathrm{i} n}(t), \hat{C}_{\mathrm{i} n}=\hat{C}_{\mathrm{i} n}(t)$ are some tensors depending on the time each of which is either an arbitrary vector for $n=1$ or an arbitrary irreducible tensor for $n>1, p_{0}=p_{0}(t)$ is the pressure at the center of the drop, which is to be found.

\subsection{Asymptotic expansion}

The coefficients are sought for in the form of the following asymptotic expansions over the parameter

$$
\alpha=\frac{9 a \mu_{\mathrm{e}} \max \left(H_{\mathrm{a}}\right)^{2}}{32 \pi \sigma_{\mathrm{s}}}
$$

$$
\begin{gathered}
\hat{M}_{\mathrm{e} n} \sim \sum_{j=0}^{\infty} \alpha^{j} \hat{M}_{\mathrm{e} n, j}, \hat{\Omega}_{\mathrm{e} n} \sim \sum_{j=1}^{\infty} \alpha^{j} \hat{\Omega}_{\mathrm{e} n, j}, \\
\hat{B}_{\mathrm{e} n} \sim \sum_{j=1}^{\infty} \alpha^{j} \hat{B}_{\mathrm{e} n, j}, \hat{C}_{\mathrm{e} n} \sim \sum_{j=1}^{\infty} \alpha^{j} \hat{C}_{\mathrm{e} n, j}, \\
\hat{M}_{\mathrm{i} n} \sim \sum_{j=0}^{\infty} \alpha^{j} \hat{M}_{\mathrm{i} n, j}, \hat{\Omega}_{\mathrm{i} n} \sim \sum_{j=1}^{\infty} \alpha^{j} \hat{\Omega}_{\mathrm{i} n, j}, \\
\hat{B}_{\mathrm{i} n} \sim \sum_{j=1}^{\infty} \alpha^{j} \hat{B}_{\mathrm{i} n, j}, \hat{C}_{\mathrm{i} n} \sim \sum_{j=1}^{\infty} \alpha^{j} \hat{C}_{\mathrm{i} n, j}, \\
p_{0} \sim \sum_{j=0}^{\infty} \alpha^{j} p_{0 j}, \hat{h}_{n} \sim \sum_{j=1}^{\infty} \alpha^{j} \hat{h}_{n, j} \text { as } \alpha \rightarrow 0 .
\end{gathered}
$$

The found nonzero terms of the asymptotic expansions are as follows

$$
\begin{gathered}
\hat{M}_{\mathrm{e} 1,0}=\vec{M}_{\mathrm{e} 1,0}=\hat{M}_{\mathrm{i} 1,0}=\vec{M}_{\mathrm{i} 1,0}=\frac{\mu_{\mathrm{i}}-\mu_{\mathrm{e}}}{\mu_{\mathrm{i}}+2 \mu_{\mathrm{e}}} a^{3} \vec{H}_{\mathrm{a}}, \\
p_{0,0}=p_{\infty}+\frac{2 \sigma_{\mathrm{s}}}{a}+\frac{3 \mu_{\mathrm{e}}}{8 \pi} \frac{\mu_{\mathrm{e}}-\mu_{\mathrm{i}}}{\mu_{\mathrm{i}}+2 \mu_{\mathrm{e}}} H_{\mathrm{a}}^{2} \\
\hat{B}_{\mathrm{e} 2,1}=\hat{B}_{\mathrm{i} 2,1}=\frac{3}{10} \frac{2 \eta_{\mathrm{e}}+\eta_{\mathrm{i}}}{\eta_{\mathrm{e}}+\eta_{\mathrm{i}}} \frac{\mathrm{d} \hat{h}_{2,1}}{\mathrm{~d} t} \\
\hat{C}_{\mathrm{e} 2,1}=\hat{C}_{\mathrm{i} 2,1}=\frac{1}{6} \frac{\mathrm{d} \hat{h}_{2,1}}{\mathrm{~d} t} \\
\vec{M}_{\mathrm{e} 1,1}=\frac{2}{5}\left(\mu_{\mathrm{e}}-\mu_{\mathrm{i}}\right) \frac{13 \mu_{\mathrm{e}}+11 \mu_{\mathrm{i}}}{\left(\mu_{\mathrm{i}}+2 \mu_{\mathrm{e}}\right)^{2}} a^{2} \vec{H}_{\mathrm{a}} \cdot \hat{h}_{2,1}^{*} \\
\vec{M}_{\mathrm{i} 1,1}=\frac{4}{5}\left(\mu_{\mathrm{e}}-\mu_{\mathrm{i}}\right) \frac{7 \mu_{\mathrm{i}}+5 \mu_{\mathrm{e}}}{\left(\mu_{\mathrm{i}}+2 \mu_{\mathrm{e}}\right)^{2}} a^{2} \vec{H}_{\mathrm{a}} \cdot \hat{h}_{2,1}^{*} \\
\hat{M}_{\mathrm{e} 3,1}=\frac{\mu_{\mathrm{i}}-\mu_{\mathrm{e}}}{\mu_{\mathrm{i}}+2 \mu_{\mathrm{e}}} \frac{5 \mu_{\mathrm{i}}-8 \mu_{\mathrm{e}}}{3 \mu_{\mathrm{i}}+4 \mu_{\mathrm{e}}} a^{4}\left[\left(\vec{H}_{\mathrm{a}} \hat{h}_{2,1}^{*}\right)^{\mathrm{S}}\right]^{\mathrm{D}},
\end{gathered}
$$



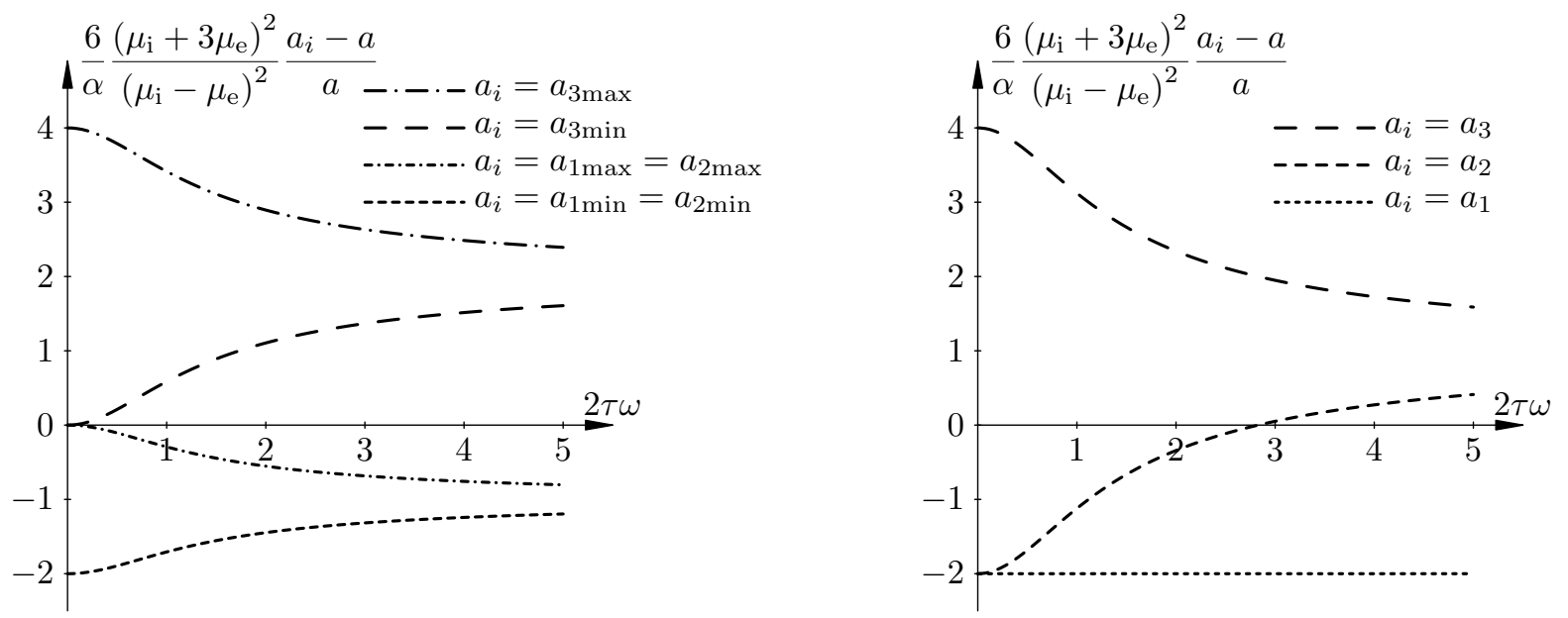

Figure 1. Steady-state oscillations of the drop: in an oscillating (left) and in a rotating (right) magnetic fields

$$
\hat{M}_{\mathrm{i} 3,1}=-4 \frac{\mu_{\mathrm{i}}-\mu_{\mathrm{e}}}{\mu_{\mathrm{i}}+2 \mu_{\mathrm{e}}} \frac{\mu_{\mathrm{i}}-\mu_{\mathrm{e}}}{3 \mu_{\mathrm{i}}+4 \mu_{\mathrm{e}}} a^{4}\left[\left(\vec{H}_{\mathrm{a}} \hat{h}_{2,1}^{*}\right)^{\mathrm{S}}\right]^{\mathrm{D}},
$$

where

$$
\left[\left(\vec{H}_{\mathrm{a}} \hat{h}_{2,1}^{*}\right)^{\mathrm{S}}\right]^{\mathrm{D}}=\left(\vec{H}_{\mathrm{a}} \hat{h}_{2,1}^{*}\right)^{\mathrm{S}}-\frac{2}{5}\left(\vec{H}_{\mathrm{a}} \cdot \hat{h}_{2,1}^{*} \hat{I}\right)^{\mathrm{S}}
$$

and $\hat{h}_{2,1}=\hat{h}_{2,1}(t)$ is a solution of the following ordinary differential equation

$$
\begin{array}{r}
\frac{\left(16 \eta_{\mathrm{e}}+19 \eta_{\mathrm{i}}\right)\left(3 \eta_{\mathrm{e}}+2 \eta_{\mathrm{i}}\right)}{10\left(\eta_{\mathrm{e}}+\eta_{\mathrm{i}}\right)} \alpha \frac{\mathrm{d} \hat{h}_{2,1}}{\mathrm{~d} t}+\frac{4 \alpha \sigma_{\mathrm{s}}}{a} \hat{h}_{2,1} \\
=\frac{9 a}{8 \pi}\left(\frac{\mu_{\mathrm{i}}-\mu_{\mathrm{e}}}{\mu_{\mathrm{i}}+2 \mu_{\mathrm{e}}}\right)^{2}\left(\vec{H}_{\mathrm{a}}^{2}-\frac{1}{3} H_{\mathrm{a}}^{2} \hat{I}\right) .
\end{array}
$$

\subsection{Steady-state oscillations}

With accuracy up to the terms of the first order, in an oscillating applied magnetic field with the intensity $\vec{H}_{\mathrm{a}}=H_{\mathrm{am}} \cos (\omega t) \vec{k}$, the drop is a prolate spheroid with the axis directed along $\vec{k}$ and with the semiaxes

$$
\begin{gathered}
a_{1}=a_{2}= \\
a\left\{1-\frac{\alpha}{6}\left(\frac{\mu_{\mathrm{i}}-\mu_{\mathrm{e}}}{\mu_{\mathrm{i}}+2 \mu_{\mathrm{e}}}\right)^{2}\left[1+\frac{\cos (2 \omega t-2 \phi)}{\sqrt{1+4 \tau^{2} \omega^{2}}}\right]\right\} \\
a_{3}=a\left\{1+\frac{\alpha}{3}\left(\frac{\mu_{\mathrm{i}}-\mu_{\mathrm{e}}}{\mu_{\mathrm{i}}+2 \mu_{\mathrm{e}}}\right)^{2}\left[1+\frac{\cos (2 \omega t-2 \phi)}{\sqrt{1+4 \tau^{2} \omega^{2}}}\right]\right\}
\end{gathered}
$$

where

$$
\begin{gathered}
\tau=\frac{\left(16 \eta_{\mathrm{e}}+19 \eta_{\mathrm{i}}\right)\left(3 \eta_{\mathrm{e}}+2 \eta_{\mathrm{i}}\right)}{10\left(\eta_{\mathrm{e}}+\eta_{\mathrm{i}}\right)^{2}} \frac{a\left(\eta_{\mathrm{e}}+\eta_{\mathrm{i}}\right)}{4 \sigma_{\mathrm{s}}}, \\
\phi=\frac{1}{2} \arctan (2 \tau \omega) .
\end{gathered}
$$

Thus, the drop performs deformational oscillations with the angular frequency $2 \omega$ and the phase lag $2 \phi$. As $\omega \rightarrow \infty$, the drop tends to take the shape of an unvarying prolate spheroid (see figure 1 left).

In a rotating applied magnetic field with the intensity $\vec{H}_{\mathrm{a}}=H_{\mathrm{am}}[\cos (\omega t) \vec{i}+\sin (\omega t) \vec{j}]$, the drop takes the shape of a tri-axial ellipsoid with the semiaxes

$$
\begin{aligned}
& a_{1}=a\left[1-\frac{\alpha}{3}\left(\frac{\mu_{\mathrm{i}}-\mu_{\mathrm{e}}}{\mu_{\mathrm{i}}+2 \mu_{\mathrm{e}}}\right)^{2}\right], \\
& a_{2}=a\left[1+\frac{\alpha}{6}\left(\frac{\mu_{\mathrm{i}}-\mu_{\mathrm{e}}}{\mu_{\mathrm{i}}+2 \mu_{\mathrm{e}}}\right)^{2}\left(1-\frac{3}{\sqrt{1+4 \tau^{2} \omega^{2}}}\right)\right] \\
& a_{3}=a\left[1+\frac{\alpha}{6}\left(\frac{\mu_{\mathrm{i}}-\mu_{\mathrm{e}}}{\mu_{\mathrm{i}}+2 \mu_{\mathrm{e}}}\right)^{2}\left(1+\frac{3}{\sqrt{1+4 \tau^{2} \omega^{2}}}\right)\right] .
\end{aligned}
$$

The ellipsoid rotates around its minor axis, directed along $\vec{k}=\vec{i} \times \vec{j}$, with the angular speed $\omega$ so that its major axis lags from $\vec{H}_{\mathrm{a}}$ by the angle $\phi$. Here, $\vec{i}, \vec{j}$, and $\vec{k}$ form a triple of orthonormal vectors. As $\omega \rightarrow \infty$, the drop tends to take the shape of an unvarying oblate spheroid (see figure 1 right).

The partial support by RFBR grants 16-01-00157 and 17-01-00037 is acknowledged.

\section{References}

[1] A. V. Lebedev, A. Engel, K. I. Morozov, and H. Bauke. Ferrofluid drops in rotating magnetic fields. New J. Phys., Vol. 5 (2003), pp. 57.1-57.20.

[2] R.E. Rosensweig, Ferrohydrodynamics (Cambridge University Press, 1985).

[3] L.D. Landau, E.M. Lifshits, The classical theory of fields (Pergamon Press, 1975).

[4] H. Lamb, Hydrodynamics (Cambridge University Press, 1932). 